\title{
Biocompatible Chitosan Nanofibers Functionalized with Silver Nanoparticles for SERS Based Detection
}

\author{
E.S. Prikhozhdenko ${ }^{a}$, E.V. Lengert ${ }^{a}$, B.V. PArakhonskiY ${ }^{a, b, c}$, D.A. Gorin ${ }^{a, d}$, \\ G.B. SuKhORUKOV ${ }^{e, f}$ AND A.M. YASHChENOK ${ }^{a, g *}$ \\ ${ }^{a}$ Remote Controlled Theranostic Systems Lab, Institute of Nanostructures and Biosystem, \\ Saratov State University, Saratov, Russia \\ ${ }^{b}$ A.V. Shubnikov Institute of Crystallography, Russian Academy of Science, Moscow, Russia \\ ${ }^{c}$ Department of Molecular Biotechnology, Ghent University, Ghent, Belgium, 9000 Ghent, Belgium \\ ${ }^{d}$ RASA Center in Tomsk, Tomsk Polytechnic University, Tomsk, Russia \\ ${ }^{e}$ Queen Mary University of London, London, UK \\ ${ }^{f}$ RASA Center in St. Petersburg, Peter the Great St. Petersburg Polytechnic University, St. Petersburg, Russia \\ ${ }^{g}$ Max Plank Institute of Colloids and Interfaces, Department of Interfaces, Potsdam, Germany \\ Electrospun chitosan nanofibrous substrates are functionalized with silver nanoparticles by reduction of silver \\ from Tollens reagent using glucose. Filling factor is estimated through developed protocol by using analysis of \\ scanning electron microscopy images. Obtained nanocomposite silver-chitosan plasmonic films display reliable \\ surface enhanced Raman scattering signal of rhodamine B with the concentration $10^{-5} \mathrm{M}$ adsorbed onto the \\ surface of functionalized substrates.
}

DOI: 10.12693 /APhysPolA.129.247

PACS: 78.67.Bf, 73.20.Mf, 81.05.Qk, 78.30.-j

\section{Introduction}

Assembly of plasmonic nanoparticles, in particular gold and silver, in arrayed nanoscale microstructures has received tremendous attention in the last years due to their collective organization leading to tunable optical properties [1]. These systems are highly desirable in a number of applications ranging from optics and electronics to biodiagnostic and medicine [2-4]. Strong environmentally sensitive light scattering caused by localized surface plasmon resonance (LSPR) of electrons at the metal surface makes plamonic nanoparticles (NPs) beneficial to develop ultrasensitive sensors, for instance based on surface enhanced Raman scattering (SERS) detection [5]. Unlike of individual nanoparticle, ensemble of NPs allow controlling over their LSPR by organizing nanoparticles in confined volume and thus tune their response to light in a predictable manner. Tuning the surface chemistry of NPs by specific ligands along with selfassembly technique can be used for the construction of hierarchical plasmonic nanostructures [6]. A template assisted approach by using planar substrates and artificial colloidal microparticles has been established as a simple and versatile method for the construction of functional $2 \mathrm{D}$ and $3 \mathrm{D}$ microstructures ranging from hollow polyelectrolyte microcapsules and plasmonic microspheres to free-standing plasmonic polymer nanofibrous films [7-

\footnotetext{
* corresponding author; e-mail: Alexey.Yashchenok@mpikg.mpg.de
}

10]. The latter is of crucial importance for the engineering of robust and reproducible SERS-active substrates. Here, we propose one-pot functionalization of biocompatible chitosan nanofibers with silver nanoparticles. By this protocol, large-scale plasmonic chitosan nanofibrous films were engineered. The quantities of silver nanoparticles in chitosan nanofiber substrates were estimated by analysis of scanning electron microcopy (SEM) images of modified nanofibrous films. Developed plasmonic nanofibers exhibited effective SERS detection of the rhodamine B of concentration $10^{-5} \mathrm{M}$ at extremely low laser intensity.

\section{Experimental section \\ 2.1. Materials}

Silver nitrate $\left(\mathrm{AgNO}_{3}\right), \mathrm{D}-(+)$-glucose $\left(\mathrm{C}_{6} \mathrm{H}_{12} \mathrm{O}_{6}\right.$, $180.16 \mathrm{Da}, \geq 99.5 \%), \mathrm{NH}_{4} \mathrm{OH}$, poly(ethylene oxide) (PEO, $1000 \mathrm{kDa}$ ), rhodamine $\mathrm{B}$ (dye content $\times 95 \%$, $479.01 \mathrm{Da})$ were purchased from Sigma-Aldrich. Chitosan (200 kDa, 80-85\% degree of deacetylation) was purchased from Joint-Stock Company "Bioprogress" (Russia). In all experiments, ultra-pure water with resistivity higher than $18.2 \mathrm{M} \Omega \mathrm{cm}$ was used.

\subsection{Synthesis of electrospinning nanofibrous membranes}

Chitosan and poly(ethylene oxide) with ratio of $93 / 7$ were prepared by mixing under vigorous stirring for $2 \mathrm{~h}$. Before that chitosan $(6 \% \mathrm{w} / \mathrm{v})$ and PEO $(1 \% \mathrm{w} / \mathrm{v})$ solutions were prepared separately by dissolving chitosan or PEO powder in $70 \%$ acetic acid. Nonwoven chitosan fiber mats were produced utilizing a needle-free laboratory device for textile electrospinning, the NanoSpider 
NS 200 (Elmarco, Czech Republic). The distance between the four-wire rotating spinning electrode and the collecting electrode was $150 \mathrm{~mm}$, the applied voltage was set to $80 \mathrm{kV}$.

\subsection{Fabrication of silver chitosan nanofibers}

The functionalization of chitosan nanofiber films with silver nanoparticles was realized by silver mirror reaction as reported in [11]. Briefly, as-prepared blank nanofiber films $\left(2 \times 1 \mathrm{~cm}^{2}\right)$ were immersed in $1 \mathrm{ml}$ of freshly prepared Tollens reagent, obtained by mixing equal volumes of $\mathrm{AgNO}_{3}(0.5 \mathrm{M})$ and ammonium hydroxide $(0.5 \mathrm{M})$ solutions in the Petri dish. Then to this mixture $1 \mathrm{ml}$ of ethanol was added (ratio $\mathrm{H}_{2} \mathrm{O}$ : alcohol was $1: 1$ ). The non-reacted products were removed and the films were washed with ethanol. $1 \mathrm{ml}$ of D-glucose $(40 \%$ water solution) was dropped to nanofiber films and left for 1 min to react with Tollens' reagent. Immediately after the adding of glucose solution color turned to white and then to red. This point indicates silver nanoparticle growth initiated by glucose. Then the chitosan nanofibers substrates decorated with silver nanoparticles were removed from the Petri dish and washed several times with clean water and ethanol.

\subsection{Characterization}

Scanning electron microscopy (SEM) images were recorded by means of a Philips XL30 electron microscope at an accelerating voltage of $3 \mathrm{kV}$. Field emission environmental electron microscopy (ESEM) was performed with a high-resolution low vacuum FEI Quanda 600 FEG instrument at an operating voltage $30 \mathrm{kV}$ with extended low-vacuum capabilities. To perform Raman measurements a confocal Raman microscope (Renishaw inVia, UK) equipped with a diode-pumped $785 \mathrm{~nm}$ NIR laser excitation $(60 \mathrm{~mW})$ controlled by a neutral optical density filter was used. The laser beam was focused through a $50 \times($ Leica N PLAN, NA $=0.5)$ microscope objective. The spectra were acquired with a thermoelectrically cooled CCD detector optimized for near IR (spectral resolution of $\left.1 \mathrm{~cm}^{-1}\right)$. All spectra were collected by using WiRE software V4.1 (Renishaw, UK) and SynchroScan was applied for processing of spectra.

\subsection{Image analysis}

Analysis of SEM images was done by using ImageJ software [12]. Selected areas of chitosan nanofiber substrates on SEM images were chosen to count the silver nanoparticles. We defined areas that are in one plane to ensure homogeneous distribution of silver nanoparticles. The filling factor was considered as particle cross-section divided by the cross-section of the polymer nanofiber film in randomly selected areas of polymer nanofiber mats. The size of the area cross-section of polymer mats is considered equal to a laser spot size $(1.8 \mu \mathrm{m})$. For each sample a minimum of five images were counted. The statistics of the filling factor was done by one way ANOVA test based on statistics of 5 images. The significant difference were set up for $p<0.05$.

\section{Result and discussions}

Silver plasmonic chitosan nanofibers substrates were obtained by silver mirror reaction using glucose as a reductant agent (Fig. 1). SEM images of functionalized chitosan nanofibers with silver nanoparticles are shown in Fig. 2. One can see that chitosan nanofibers are intact after treatment with Tollens' reagent. In addition, silver nanoparticles are distributed almost uniformly in nanofibers. However, some aggregates also appeared in irregularities and intersections of nanofibers. In order to estimate the amount of silver nanoparticles in the films, we apply SEM image analysis. We find that the distribution of silver nanoparticles in chitosan nanofibrous films changes with increase of the reduction cycles. Oneway ANOVA test was used for the statistics of the filling factor. It was found that no difference in number of silver nanoparticles for the first $(47.4 \pm 6.4 \%)$ and double $(49.2 \pm 1.5 \%)$ reduction. We predict that nanofibers films become mainly saturated by the silver nanoparticles in first reduction. For this reason, only one cycle of synthesis is enough and following silver reduction does not improve the scaffold properties. blank nanofibers
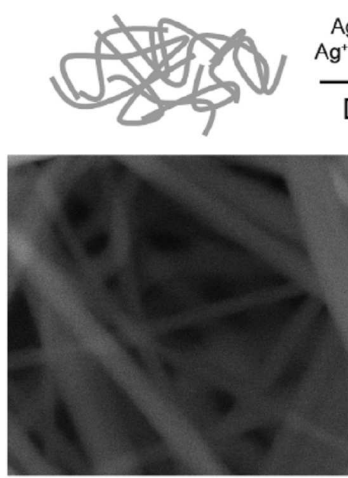

functionalized nanofibers
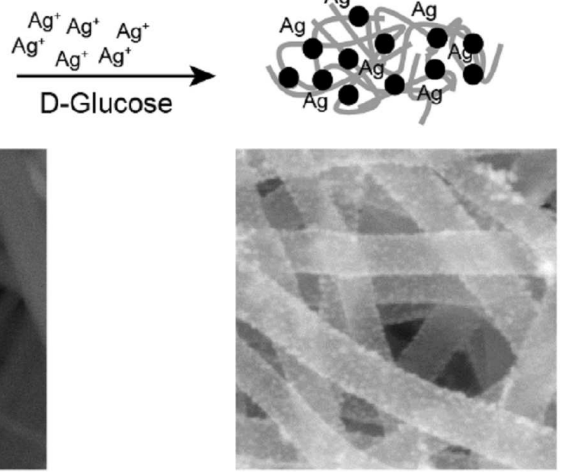

Fig. 1. Schematics showing preparation of electrospun chitosan nanofiber substrates functionalized with silver nanoparticles by silver mirror reaction.

The SERS capability of silver chitosan nanofibers was tested by measuring rhodamine $\mathrm{B}$ (RhoB) as the model molecule. Prior to SERS measurements, RhoB was dissolved in water with the concentration $10^{-3} \mathrm{M}$ and Raman reference spectrum was acquired (Fig. 3, black curve). Silver chitosan nanofibers were incubated with concentration of RhoB $10^{-5} \mathrm{M}$ for $1 \mathrm{~h}$ to ensure adsorption of chromophore molecules onto the surface of nanofibers. The SERS measurements were acquired in five randomly selected points in nanofiber films. Several peaks centered at $737 \mathrm{~cm}^{-1}$ (aromatic ring in-plane bending), $1203 \mathrm{~cm}^{-1}$ (C-C stretching) at $1281 \mathrm{~cm}^{-1}$ (C$\mathrm{O}-\mathrm{C}$ stretching) and at $1507 \mathrm{~cm}^{-1}$ (aromatic stretching of dye) are detected for both prepared substrates. However, higher SERS intensity was found for the samples prepared in the first reduction (Fig. 3a). This can be 

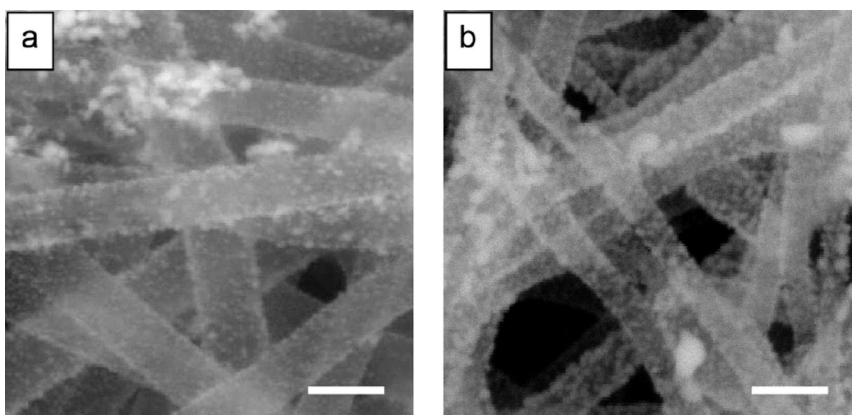

Fig. 2. SEM images of chitosan nanofibers functionalized with silver nanoparticles obtained through the initiation of the growth of silver nanoparticles by adding D-glucose. (a) Silver chitosan nanofibers prepared in the first reduction, (b) the same samples obtained in the second reduction. The scale bars correspond to $500 \mathrm{~nm}$.

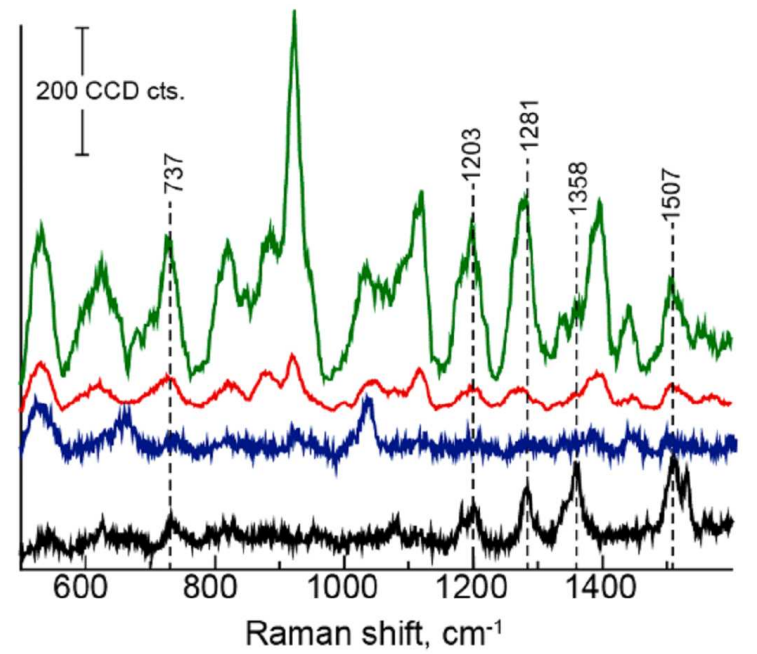

Fig. 3. SERS spectra of Rhodamine B taken from chitosan nanofibers functionalized with silver nanoparticles. Green spectrum corresponds double reduction, while red curve single one. Black spectrum is Raman spectrum of water solution of rhodamine $B\left(10^{-3} \mathrm{M}\right)$. Blue spectrum represents Raman spectrum acquired for blank chitosan nanofibers. CCD count given only for SERS spectra. All SERS spectra were acquired with laser wavelength of $785 \mathrm{~nm}$ through $50 \times$ air objective at a power of $0.06 \mathrm{~mW}$.

attributed to the shift of the surface plasmon peak to the red part of the visible spectrum in the case of second reduction [13]. In addition, this can be induced by a decrease of number of "hot spots" due to the overgrowth of silver nanoparticles in chitosan nanofibrous films. It is worth to mention that chitosan vibrational peaks observed in SERS spectra do not overlap with that for rhodamine and peaks from rhodamine can be easy resolved.

\section{Conclusions}

Biocompatible chitosan free-standing substrates have been obtained by electrospinning technique and functionalized with silver nanoparticles by applying silver mirror reaction. This approach allowed us to reduce the number of intermediate steps for the preparation of plasmonic nanofibers. We found that the number of reduction cycles do not influence on the distribution of silver nanoparticles in chitosan substrates, which means that the chitosan reaches the saturation by silver nanoparticles and new formatted particles did not attach to the surface. This plasmonic chitosan nanofibrous substrate displays effective SERS detection of the standard rhodamine B of concentration $10^{-5} \mathrm{M}$. The approach shown here would helpful in the development of efficient and scalable SERSactive substrates along with the control of their optical properties.

\section{Acknowledgments}

The authors thanks to V.S. Atkin for SEM measurement. This work was supported by Government of the Russian Federation (grant No. 14.Z50.31.0004 to support scientific research projects implemented under the supervision of leading scientists at Russian institutions and Russian institutions of higher education). We acknowledge RFBR research project No. 15-29-01172 ofi_m, for support. B.P. acknowledge the FWO for postdoctoral scholarship.

\section{References}

[1] A. Engelko, H. Bluhm, J. Appl. Phys. 95, 5828 (2004); A. Klinkova, R.M. Choueiri, E. Kumacheva, Chem. Soc. Rev. 43, 3976 (2014).

[2] B. Ai, Y. Yu, H. Möhwald, G. Zhang, Adv. Opt. Mater. 1, 724 (2013).

[3] H.A. Atwater, A. Polman, Nat. Mater. 9, 205 (2010).1

[4] N. Khlebtsov, V. Bogatyrev, L. Dykman, B. Khlebtsov, S. Staroverov, A. Shirokov, L. Matora, V. Khanadeev, T. Pylaev, N. Tsyganova, G. Terentyuk, Theranostics 3, 167 (2013).

[5] H. Wei, H.X. Hot, Nanoscale 5, 10794 (2013).

[6] S. Srivastava, N.A. Kotov, Soft Matter 5, 1146 (2009).

[7] Q. Zhao, B. Han, Z. Wang, C. Gao, C. Peng, J.C. Shen, Nanomedicine 3, 63 (2007).

[8] A. Yashchenok, B. Parakhonskiy, S. Donatan, D. Kohler, A. Skirtach, H. Möhwald, J. Mater. Chem. B 1, 1223 (2013).

[9] X. Yan, J. Li, H. Möhwald, Adv. Mater. 24, 2663 (2012).

[10] A.M. Yashchenok, D. Borisova, B.V. Parakhonskiy, A. Masic, B. Pinchasik, H. Möhwald, A.G. Skirtach, Ann. Phys. 524, 723 (2012); A.N. Severyukhina, B.V. Parakhonskiy, E.S. Prikhozhdenko, D.A. Gorin, G.B. Sukhorukov, H. Moehwald, A.M. Yashchenok, ACS Appl. Mater. Interfaces 7, 15466 (2015).

[11] T. Bukreeva, I.V. Marchenko, B.V. Parakhonskiy, Y.V. Grigorev, Colloid J. 71, 596 (2009).

[12] http://rsb.info.nih.gov/ij/.

[13] N. Pazos-Perez, C.S. Wagner, J.M. Romo-Herrera, L.M. Liz-Marzán, F.J. García De Abajo, A. Wittemann, A. Fery, R.A. Alvarez-Puebla, Angew. Chemie - Int. Ed. 51, 12688 (2012). 